\title{
TENTATIVES DE RAPPROCHEMENT \\ FRANCO-ALLEMAND \\ LA QUESTION FRANCO-ALLEMANDE \\ À LA NOUVELLE REVUE DE HONGRIE (JUIN 1932 - DÉCEMBRE 1934)
}

\author{
HENRI DE MONTETY \\ Rédacteur aux Hungarian Studies \\ hmontety@gmail.com
}

\begin{abstract}
Frustrée par les frontières imposées lors du traités de paix de 1920, la Hongrie a tenté, entre les deux guerres, d'agir sur les esprits afin d'obtenir la modification pacifique de l'ordre des relations internationales. La Nouvelle Revue de Hongrie, rédigée de manière indépendante, mais de concert avec le ministère des Affaires étrangères hongrois, entrait dans le cadre de cette diplomatie non officielle en s'adressant aux élites francophones du monde entier. Tout en présentant la culture hongroise, elle abordait des sujets de l'actualité internationale, de manière parfois complexe et ambitieuse. Une dizaine d'articles de la revue, publiés entre 1932 et 1934, montrent l'ambition (sans doute démesurée) de contribuer à la réconciliation franco-allemande.
\end{abstract}

Mots-clefs : Relations franco-allemandes, années trente, nationalisme, pacifisme, Europe

La Nouvelle Revue de Hongrie (NRH) parut entre 1932 et 1944. Sous la conduite spirituelle du comte István Bethlen, elle était co-dirigée par l'ancien diplomate et publiciste polygraphe Georges Ottlik et Joseph Balogh, juif converti au catholicisme, à la fois traducteur de Saint Augustin, publiciste infatigable et homme d'affaires.

Instrument quasi-officiel de la propagande hongroise, la $N R H$ était aussi une revue internationale de qualité, préparée dans sa forme comme dans son fond pour influencer le public français et francophone qui était, soit par ignorance, soit par préjugés, spontanément peu enclin à entendre les arguments hongrois. Tout en présentant le meilleur de la production culturelle hongroise ancienne et surtout contemporaine, son objectif était d'établir dans les esprits la solidarité entre les revendications hongroises et la résolution des problèmes internationaux (autrement dit: "la révision, c'est la paix"). Au début des années trente, la revue organisa fréquemment des controverses où de grandes plumes étrangères étaient invitées à s'exprimer sur une question d'actualité ; l'espoir était que le point de vue et les intérêts hongrois se dégageassent indirectement du débat comme facteurs de réconciliation et d'entente durable. 


\section{Les relations franco-allemandes comme fondement de la politique européenne}

En ce temps-là, le "tête-à-tête" avec l'Allemagne était (déjà) un aspect stratégique, non seulement pour la France, mais aussi pour l'Europe. On se tenait au chevet de la relation franco-allemande comme à celui de la destinée de l'Europe voire de la civilisation. Mentionnons, parmi les guérisseurs, l'ambassadeur des États-Unis ${ }^{1}$ ou les "Décades" de Pontigny ${ }^{2}$, de même que la gauche socialiste et radicale ou les milieux économiques favorables à l'union douanière (Loucheur, Le Trocquer, le Comité franco-allemand d'information et de documentation), mais aussi une cohorte de catholiques de gauche et de syndicalistes chrétiens ${ }^{3}$, entre autres, et des publicistes plus ou moins conservateurs et hétérodoxes comme Wladimir d'Ormesson et Jean de Pange. Dans son journal, ce dernier allait écrire combien tous ces projets n'avaient été qu'illusion ${ }^{4}$.

Articles de la Nouvelle revue de Hongrie consacrés à la réconciliation franco-allemande

Wladimir d'Ormesson (Le Figaro), « La France devant le problème allemand » (juin 1932)

Friedrich Sieburg (Frankfurter Zeitung), «La politique du rapprochement sans phrase »

(sept. 1932)

Otto Hoetzsche (professeur d'université), «L'Allemagne devant le problème français » (sept. 1932)

Robert d'Harcourt (Institut catholique de Paris), « Le problème franco-allemand » (oct. 1932)

René Dupuis (jeune publiciste), «"Statisme” et "Dynamisme”. Le nationalisme français devant le problème de la sécurité et des relations franco-allemandes » (oct. 1932 - en fin de numéro)

Wladimir d'Ormesson, «Égalité - Confiance » (nov. 1932)

Friedrich Sieburg, « Où en est la politique franco-allemande ?» (jan. 1934)

Bertrand de Jouvenel (jeune publiciste radical), « Réflexions sur les rapports franco-allemands » (fév. 1934)

$\mathrm{C}^{\text {te }}$ Jean de Pange (publiciste), « Les relations franco-allemandes » (oct. 1934)

Richard Jügler (Berliner Börser-Zeitung), « Réponse à Monsieur le comte de Pange » (déc. 1934)

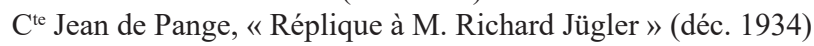

Georges Roux (jeune publiciste, avocat), « Germanisme et latinité » (nov. 1935)

E. d'Astier de la Vigerie (publiciste), « Le problème danubien et les relations franco-allemandes » (sept. 1937)

Jean de Pange, «Qu'est-ce qu'une frontière ? La frontière franco-allemande » (déc. 1937)

Richard von Kühlmann (Sous-secrétaire d'État à la Wilhelmstrasse), «L'Allemagne et la France » (fév. 1938)

André Beauguitte (député), « Réflexions sur la déclaration franco-allemande » (jan. 1939)

Dom Germain Morin OSB, « Les deux ailes de l'Occident » (août 1940)

Une illusion de plus était peu de chose pour les Hongrois, qui emboîtèrent le pas avec enthousiasme. (D'ailleurs, l'illusion portait sans doute sur la possibilité du rapprochement franco-allemand, plus que sur sa nécessité.) C'est ainsi que 
dès ses débuts ${ }^{5}$, la $N R H$ obtint du célèbre Wladimir d'Ormesson la livraison d'un article sur « La France devant le problème allemand » (juin 1932). Peu après, l'Allemand Otto Hoetzsche répondait naturellement par « L'Allemagne devant le problème français » (septembre 1932). Dans le même numéro, Friedrich Sieburg se contentait d'une sobre « Politique du rapprochement sans phrase ». Trois nouveaux articles parurent jusqu'à la fin de l'année, c'est-à-dire jusqu'à la prise de pouvoir par Hitler en janvier 1933 qui suspendit toute discussion pendant un an. Néanmoins, en 1934, le débat allait reprendre de plus belle (cinq articles), mais ce fut un feu de paille.

\section{Incompréhension et animosité (1932 : d'Ormesson, Hoetzsche, Sieburg, d'Harcourt, Dupuis)}

Comme base de départ du dialogue, en même temps que signe de sa difficulté, tous les protagonistes admettaient que les situations intérieures respectives, en France et en Allemagne, étaient incomparables. Pire, d'après Wladimir d'Ormesson, les deux pays étaient perpétuellement dans un rapport asynchrone ; ainsi, la Chambre progressiste fraîchement élue en France rencontrait en face d'elle une Allemagne plongée dans le militarisme ${ }^{6}$. Il ajoutait que l'Allemagne se distinguait aussi par l'inquiétante proportion de son prolétariat. Hoetzsche partageait cet avis, estimant que la population allemande était aux 3/4 prolétarisée, mais il affirmait aussi que la crise allait sans doute finir par toucher la France, elle aussi, malgré le « sentiment d'avoir fait tout le nécessaire » qui transparaissait dans les propos de Wladimir d'Ormesson. Friedrich Sieburg insistait, quant à lui, sur la différence qui séparait la torpeur française et le dynamisme de la révolution allemande. Justement, d'après Hoetzsche, nul Français n'était capable de mesurer le courant irrésistible qui était en train de naître en Allemagne (il écrivait cela en septembre 1932). Le jeune René Dupuis, qui fut admis à s'exprimer à la fin du numéro d'octobre, affirmait qu'il n'avait pas « l'intention d'intervenir dans le débat en cours, mais [seulement] de définir la conception de la sécurité chez les nationalistes français. » Aussi expliquait-il la rigidité du nationalisme français (i.e. l'intransigeance sur les questions de la révision des traités et du désarmement) par son caractère essentiellement défensif. Néanmoins, il ne désespérait pas que la situation changeât.

Les messieurs qui débattaient en première page étaient moins optimistes, car, selon eux, la différence des situations nationales respectives avait pour conséquence une incompréhension totale. Wladimir d'Ormesson se sentait obligé d'affirmer préventivement que l'Allemagne ne devait pas espérer de faiblesse de la part de la gauche française, pour laquelle il s'agissait tout simplement de sauver sa politique (donc son avenir électoral). Sieburg convenait que le fossé était 
désormais profond, car, en Allemagne, la guerre avait été déclarée au raisonnable (donc à tout ce qui était français), considéré comme ennemi irréductible des valeurs nationales. De son côté, Robert d'Harcourt affirmait que les Allemands ignoraient tout des « réflexes profonds » français, liés aux dures années de guerre et au saccage d'une partie du territoire national ${ }^{7}$. De la part de personnalités œuvrant pour le rapprochement des peuples, le constat paraissait, dans l'ensemble, bien amer et peu optimiste. De fait, c'était pire encore, car l'incompréhension est sœur de la méfiance et cousine de l'animosité. La France est inquiète, écrivait ainsi Wladimir d'Ormesson. Encore ? s'étonnait Sieburg - voici venue l'heure de la leçon de morale ! Donnée, de plus, par une France qui a pour coutume de déguiser sa politique extérieure en grands principes universels (plan Tardieu, politique de Briand), par une France qui n'a jamais « de son bon gré, fait une concession à 1'Allemagne ». Hoetzsche renchérissait en soulignant que toute la politique internationale actuelle avait pour fondement l'inégalité : pas un seul Allemand dans l'organigramme de la $\mathrm{SdN}$, hégémonie de la langue française dans tous les services de l'organisation. Et surtout : le problème de l'armement, qui n'était qu'un prétexte au maintien du statu quo. Face à ces revendications, Robert d'Harcourt affirmait souscrire entièrement à l'égalité morale entre les peuples, mais cela ne devait impliquer en aucune manière « la radiation sommaire et surtout unilatérale des inégalités d'ordre pratique, conséquence d'une guerre dont l'un des peuples [était] sorti victorieux et l'autre vaincu. » Distinction digne d'un théologien thomiste, mais qui devait laisser froids ses interlocuteurs germaniques. De plus, d'Harcourt renvoyait à l'Allemagne l'accusation, affirmant qu'elle ne respectait pas la parité morale, quand, drapée dans son prétendu bon droit, sa presse unanime vilipendait quotidiennement la France (buveuse de sang, etc...). Ce point fut repris par d'Ormesson dans son article de novembre, qui clôturait le débat pour l'année, où il réitéra son diagnostic : «Ce qui est vrai, c'est que la France n'a pas confiance en l'Allemagne », car cette dernière n'a pas su apprécier les gestes sincères de la France (évacuation de la Rhénanie en 1930, renoncement aux réparations, soutien financier). Vladimir d'Ormesson ne craignait pas d'affirmer « devant Dieu » que ceux qui parlaient d'hypocrisie à propos de la France se trompaient. Bref. N'y avait-il vraiment rien à faire ? Rien ne se fera tant que les journalistes n'auront pas pris conscience de leurs responsabilités ! martelait Robert d'Harcourt.

Sieburg avertissait que la politique des « petits pas » n'avait aucun avenir (un jour les réparations, un jour le désarmement, puis la Sarre, puis les frontières de l'Est, les responsabilités, etc...). Seule une approche globale, traitant l'ensemble comme un «tout indivis » pourrait résoudre le problème des relations franco-allemandes. (Certes, Hitler, quant à lui, allait justement pratiquer, avec un certain succès, la politique des "petits pas".) En guise de synthèse démontrant implicitement le caractère incantatoire et contradictoire du rapprochement, Hoetzsche disait : égalité, confiance. Mais d'Ormesson disait : confiance, égalité. 


\section{La "clarification" hitlérienne (1934 : Sieburg, Gooch, Jouvenel, Pange, Jügler)}

Le 31 janvier 1933, Hitler accédait au pouvoir en Allemagne. La NRH suspendit le débat. Décision tactique ; celui-ci allait reprendre dès l'année suivante. En janvier 1934, Friedrich Sieburg, seul rescapé de l'année 1932, donnait naturellement pour titre à son article : «Où en est la politique franco-allemande ? » Fidèle à son style volontariste, il mêlait habilement ses désirs à l'analyse des réalités, ellemême drapée dans un mystère insondable. Malgré les réticences françaises, écrivait-il, « comme poussées par des forces mystérieuses, l'Allemagne et la France se trouvent journellement un peu plus rapprochées l'une de l'autre. » Paradoxalement, ces « forces mystérieuses » achevaient le processus de "clarification" dont on pouvait déjà observer les prémisses en 1932. Hitler avait définitivement réuni toute l'Allemagne dans son poing et, comme le soulignait Sieburg, il n'était plus question de distinguer, à la manière française, une bonne et sage Allemagne et une turbulente et agressive Allemagne. Il affirmait que son pays voulait la paix, en s'appuyant sur deux arguments : d'une part, la paix était nécessaire afin que pût s'exprimer librement l'idéal national ; d'autre part, le goût actuel pour les uniformes et les défilés n'avait rien de commun avec le vieux militarisme allemand, car l'Allemagne socialiste avait rejeté l'impérialisme bourgeois pour se consacrer au soin de son corps social. La jeunesse française, écrivait Sieburg, sympathisait avec l'ambition allemande de « se frayer un chemin vers l'avenir », même si elle n'appréciait pas certains aspects du socialisme national qu'elle trouvait parfois « dur » et « cruel ». Mais, selon lui, deux civilisations entièrement différentes pouvaient cohabiter côte à côte, dans l'égalité réciproque (toujours l'égalité, toujours la question de l'armement !). Sieburg réitérait que le but de l'Allemagne n'était pas de « changer la carte de l'Europe, mais de créer une atmosphère qui permette le retour de la confiance. »

"Smoke and mirrors" ! eût pu répliquer George Peabody Gooch", auteur d'une analyse des relations anglo-allemandes en février 1934, dont les restes de confiance venaient d'être anéantis par l'avènement d'Hitler. Dans son article, Gooch s'appuyait sur la lecture de Mein Kampf, dont le programme était manifestement en voie d'application et sur lequel le journaliste anglais donnait des détails glaçants. Était-ce la fin du débat? Non, car dans le même numéro de février, le jeune radical français, Bertrand de Jouvenel, reconnaissait qu'une explication entre la France et l'Allemagne était encore possible et nécessaire. En guise de bonne volonté, il proposait un parallèle audacieux entre l'Allemagne de Weimar et la France d'après 1815, poussant le (sur)réalisme jusqu'à comparer les déclarations pacifistes d'Hitler à celles de Lamartine en 1848. Il affirmait aussi sympathiser avec la frustration des Allemands face à la « vanité » française affichée dans le cadre de la $\mathrm{SdN}$ et soulignait avoir lui-même engagé, à Berlin, avec de jeunes 
Hitlériens « un dialogue fondé sur le principe de ne pas faire de jugement de valeur sur des régimes qui reposent sur des conditions locales. » Dans cet ordre d'idée, il considérait l'Europe centrale comme relevant de la zone d'expansion germanique, à condition que celle-ci fût de nature pacifique. Finalement, selon lui, le seul point sur lequel un certain contrôle était légitime (mais sous quelle forme ?), c'était que le « socialisme national » ne devînt pas « socialisme nationaliste ». Pour Jouvenel, jeune intellectuel pacifiste, le nationalisme, c'était la guerre.

Le lecteur de la $N R H$ eut pendant de long mois le loisir de méditer sur ces deux opinions antagonistes (celle de Jouvenel et celle de Gooch), car l'article suivant consacré aux relations franco-allemandes ne parut qu'à la fin de l'année 1934, à l'occasion d'une controverse spécifique entre Jean de Pange et Richard Jügler qui acheva de mettre en évidence l'ampleur des divergences. En octobre, le Français proposait un panorama historique de la naissance du concept honni de nationalité, envisagé comme la conjonction monstrueuse du sentiment national d'origine française et des réflexions des philologues et juristes allemands de la fin $\mathrm{du} \mathrm{XIX}^{\mathrm{e}}$ siècle, dont la conclusion était que tous les locuteurs d'une même langue devaient ressortir du même État. Jean de Pange, lorrain de toujours et Alsacien d'occasion (il vécut à Strasbourg après la guerre), ne pouvait souscrire à cette philosophie. Au contraire, selon lui, la "double nationalité" était une richesse (indispensable), un pont entre les cultures ; c'est ainsi que l'Alsace devait montrer, en France, « le meilleur de la germanité ». Une transposition géopolitique encore plus ambitieuse était la proposition de séparer de la Prusse les terres allemandes catholiques, sous la forme d'une confédération des États germaniques du sud et de l'Autriche, ou au moins d'un État rhénan autonome dans le cadre du Reich (la première thèse avait été en vogue dans les milieux catholiques au début des années vingt - Jean de Pange l'attribuait au cercle du $\mathrm{M}^{\text {al }}$ Lyautey). Mais, d'une part, Pange laissait planer une certaine ambiguïté sur la situation précise de cette partie séparée : devait-elle être nécessairement autonome ou éventuellement indépendante ? (Richard Jügler ne manqua pas de le souligner dans sa réponse publiée en décembre.) D'autre part, il semble que l'Alsace n'était pas appelée à en faire partie. Ce qui, pour les Allemands, même les plus souples, risquait de provoquer l'incompréhension.

Que disait encore Jügler dans sa réponse ? Il réfutait en bloc la leçon d'histoire du comte de Pange. D'après lui, l'expansionnisme français remontait à un passé plus lointain que les seules guerres napoléoniennes. En outre, c'était bien la France et non l'Allemagne qui s'était tenue sur le pied de guerre jusqu'en 1914. En fait, Jügler répondait plus en qualité d'allemand qu'en tant que nazi. Et il se contenta de réfuter brièvement les atrocités attribuées à l'Allemagne hitlérienne, sans même insister sur les supposés bienfaits du programme politique en cours. De son côté, Jean de Pange était près à battre sa coulpe au nom de la France. 
Au moins sur deux points complémentaires. C'était, disait-il, l'occupation de la Ruhr par l'armée française (en 1923) qui avait conduit au développement du racisme en Allemagne. Quant au pacifisme briandiste (que Jean de Pange était pourtant volontiers accusé d'embrasser), il avait eu, selon lui, l'objectif irréaliste d'organiser la paix sur des « bases verbales » au moment où toute l'Europe produisait des armes.

Tout en condamnant la barbarie du racisme, Pange se montrait prêt à faire l'inventaire du nazisme, cet « extraordinaire amalgame de doctrine que nous apporte le national-socialisme » au sein duquel il fallait en particulier « rendre justice à l'idéalisme avec lequel Darré ${ }^{9}$ [s'efforçait] de reconstituer une noblesse du sol et de la terre. » Mais cela ne servit à rien ; Jügler dénonça tout d'un trait ce qu'il semblait considérer comme une banale attaque anti-allemande. C'est pourquoi, dans sa réplique à la réponse de Jügler, Jean de Pange allait souligner son propre isolement : « M. Charles Maurras me reproche constamment, dans l'Action française, mes illusions sur l'Allemagne » déplorait-il. « Je suis tenté de lui donner raison quand je vois comment ma bonne volonté est interprétée par certains Allemands. »

Voilà comment s'achevait, en décembre 1934, le dialogue franco-allemand commencé en juin 1932 : sur une impasse ${ }^{10}$. La $N R H$ pouvait s'enorgueillir, en tant qu'organe de presse d'un petit pays humilié, d'avoir obtenu la contribution de grandes plumes européennes sur un sujet aussi sérieux. Mais, dans le fond, la controverse n'avait servi qu'à mettre en évidence les difficultés, voire l'impossibilité du dialogue franco-allemand, avant comme après l'avènement d'Hitler.

\section{Notes}

1 William Bullit, ambassadeur des États-Unis en France, considérait la réconciliation franco-allemande comme une tâche prioritaire, tout en constatant que les Britanniques, les Italiens et les Russes y étaient tous opposés. Orville H. Bullit, For the President. Personal and secret, 1973, pp. 184-189, cité dans J.-B. Duroselle, Politique étrangère de la France. La décadence (19321939), Point-Histoire, p. 313

2 La réconciliation franco-allemande fut un thème récurrent des Décades de Pontigny, réunissant sous la houlette de Paul Desjardins (1859-1940) des personnalités et des jeunes intellectuels du monde politique, philosophique et littéraire entre 1922 et 1930. François Chaubet, Histoire intellectuelle de l'Entre-deux-guerres. Culture et politique, Paris, Nouveau monde, 2006, p. 129

3 Kövics Emma, Az Európa egység kérdése és Németország, 1919-1933 [La question de 1'union européenne et l'Allemagne, 1919-1933], Akadémiai Kiadó, Budapest, 2002, p. 148

4 « Toutes les entreprises pleines de promesses auxquelles j'ai cru, la politique rhénane, la politique sarroise, la politique alsacienne, et enfin le rapprochement intellectuel franco-allemand, ont échoué lamentablement. [...] Cette expérience pourrait assez bien être décrite sous forme romanesque. » Jean de Pange, Journal, 1967, t. II (1931-1933), p. 306, cité dans J.B. Duroselle, op. cit.., p. 64

5 La Nouvelle Revue de Hongrie (1932-1944) a succédé à la Revue de Hongrie (1908-1931). 
6 Hindenburg venait de renvoyer Brüning (en mai 1932).

7 « Nous éprouvons une sorte d'inconvenance dans l'expéditive rondeur avec laquelle nos voisins nous pressent d'oublier nos plaies. » (Robert d'Harcourt, " Le problème franco-allemand ", Nouvelle Revue de Hongrie, octobre 1932, p. 216) R. d'Harcourt regrettait aussi que peu d'Allemands eussent, après la guerre, fait le pèlerinage sur les terres dévastées du nord.

8 G.P. Gooch, « Relations anglo-allemandes », Nouvelle Revue de Hongrie, février 1934, pp. 107 113

9 Walther Darré (1895-1953), membre du parti nazi depuis 1929, auteur de Neuadel aus Blut und Boden (1930) publié en France en 1939 (Nouvelle noblesse du sang et du sol). Fondateur en 1931 du Bureau de la race et du peuplement (Rasse- und Siedlungshauptamt), puis ministre de l'Agriculture jusqu'en 1942, il contribua à la doctrine nazie dans le domaine de l'expansion géographique (Lebensraum et Drang nach Osten) et de la création d'une aristocratie germanique fondée sur la reproduction sélective et enracinée sur le sol allemand.

10 Même résultat dans les coulisses. Balogh avait demandé à son ami attaché de presse de la Légation de Hongrie à Berlin de lui trouver un contradicteur pour l'article de Pange. (« Tu connais la direction et le style de notre revue : pas de polémique, mais des articles apaisés, courtois, d'un haut niveau "européen" et au-dessus de tous intérêts particuliers, et qui puisse aussi entrer dans le cadre de la propagande officielle. ») On trouva Jügler. Mais les autorités allemandes refusèrent de donner toute publicité en Allemagne, tant à l'article de Pange qu'à la réponse de Jügler. Il ne restait au rédacteur en chef de la $N R H$ qu'à déplorer ce « manque d'objectivité ». Correspondance Jozsef Balogh - Camillo Haubert, octobre à décembre 1934 (Országos Széchenyi Könyvtár. Kézirattár. Fond Balogh 1/1374) 